\title{
High-efficient up-conversion of photoluminescence in CdSe quantum dots grown in ZnSe matrix
}

\author{
V.V. Strelchuk', M.Ya. Valakh'1, M.V. Vuychik ${ }^{1}$, S.V. Ivanov ${ }^{2}$, P.S. Kop'ev ${ }^{2}$, T.V. Shubina ${ }^{2}$ \\ ${ }^{\prime}$ Institute of Semiconductor Physics, NAS of Ukraine, 03028 Kyiv, Ukraine \\ ${ }^{2}$ Ioffe Physico-Technical Institute, RAS, 194021 St.-Petersburg, Russia \\ Phone: +38(044) 265 8550; fax: +38(044)2658342,e-mail:valakh@isp.kiev.ua
}

\begin{abstract}
The intensive up-conversion photoluminescence at low temperatures in $\mathrm{CdSe} / \mathrm{ZnSe}$ structures with single CdSe inserts of the nominal thickness 1.5 monolayer was observed. Excitation power dependensies show a nearly quadratic character up-converted photoluminescence signal from CdSe quantum dots. Up-conversion photoluminescence mechanism was interpreted on the basis of a non-linear process of two-step two-photon absorption through deep defect states including cation vacancies localized at the interface of quantum dots. This observation of photoluminescence up-conversion demonstrates the influence of quantum dot environment on its properties.
\end{abstract}

Keywords: CdSe quantum dots, up-conversion photoluminescence

Paper received 25.10.02; accepted for publication 17.12.02.

\section{Introduction}

In recent years the fabrication of high quality self-organized quantum dots (QDs) becomes the major topic in semiconductor researches. CdSe/ZnSe quantum nanostructures with self-assembled QDs are of considerable interest as they offer the possibility for low-threshold-current optoelectronic devices operating in the green spectral range. As reported earlier [1-4], in CdSe formation of $\mathrm{QD}$ is more complicated as compared with $\mathrm{Ge} / \mathrm{Si}$ and InAs/GaAs QDs structures and is not restricted only to an abrupt 2D-3D threshold transition which is peculiar to the Stranski-Krastanov growth mode. The transformation becomes more complex due to the interdiffusion processes at $\mathrm{CdSe} / \mathrm{ZnSe}$ interfaces and $\mathrm{Cd}$ segregation. These processes are probably accelerated by a nonuniform strain distribution and nonequilibrium growth conditions which produce anomalously high density of cation vacancies. These defects are most likely expected to serve as a channel of nonradiactive recombination of carriers, and therefore they diminish the effective emission of QDs. In the photoluminescence (PL), these defects act as a traps for the photoexcited electrons and holes. The lifetime of the carrier trapped to the defect may be very long, and, in principle, it becomes possible to release trapped carriers by means of intra-bandgap $\left(h v_{e x c}<E_{g}\right.$, where $E_{g}$ is the bandgap of CdSe QDs) optical excitation. Low-temperature up-conversion photoluminescence (UPL), or antiStokes PL at extremely low excitation intensity was observed in semiconductor heterojunctions, quantum wells and QDs [5-8]. In most of these experiments, UPL was caused by radiative recombination in the wide-bandgap material.

In this paper, we report on the experimental observation of the intensive UPL at low-temperature and low excitation intensity in $\mathrm{CdSe} / \mathrm{ZnSe}$ self assembled QDs, when the optical excitation energy is lower than the energy of the QD ground state.

\section{Experimental set-up}

Epitaxial $\mathrm{CdSe} / \mathrm{ZnSe}$ structures were grown pseudomorphically by the migration-enhanced epitaxy (MEE) method on $\mathrm{GaAs}(001)$ substrates at $280-300^{\circ} \mathrm{C}$ [9]. After the standard procedure of the initiation of the $\mathrm{ZnSe}$ growth on the GaAs in the regime of the enhanced atomic migration, the $\mathrm{ZnSe}$ buffer layer $(80 \mathrm{~nm})$ was deposited. CdSe epilayers of nominal thickness of 1.5 monolayer 


\section{V.V. Strelchuk: High-efficient up-conversion of ...}

(ML) were deposited by MEE. The nominal thickness of the CdSe layer was controlled by the number of epitaxial cycles per run, with $0.3 \mathrm{ML}$ being the CdSe nominal thickness for each cycle; the interruption after every cycle lasted for $10 \mathrm{~s}$. The final step of the growth process was the deposition of the $\mathrm{ZnSe}$ covering layer of $20 \mathrm{~nm}$ thickness.

PL spectra were measured using the DFS-24 spectrometer with a photon-counting system. Different lines of $\mathrm{Ar}^{+}$-laser were used for excitation.

\section{Results and discussion}

Fig. 1 shows the PL spectra $(90 \mathrm{~K})$ of $\mathrm{CdSe} / \mathrm{ZnSe}$ heterostructure with a single CdSe insert of nominal thickness of 1.5 ML. In the Stokes range (Fig. 1(a)) the intensive band at $\sim 2.564 \mathrm{eV}$ with linewidth $\sim 35 \mathrm{meV}$ is observed. The position of the maximum of this band and its low-energy asymmetry agree with the recent results [10] and are interpreted as simultaneous contribution to the spectrum of confined excitons from $\mathrm{Cd}_{\mathrm{x}} \mathrm{Zn}_{1-\mathrm{x}} \mathrm{Se} 2 \mathrm{D}$-layer (the high-energy side) and of localized $0 \mathrm{D}$ excitons in the small intra-layer Cd-rich islands (the low-energy side).

When the excitation quantum energy is lower than the exciton energy maximum (Fig. 1(c,d)), an up-conversion emission is observed. The mechanism of UPL excitation can be interpreted on the basis of the model proposed for UPL in InAs and InP QDs [5, 8], namely, the non-linear process of the two-step two-photon absorption (TS-TPA) of the excitation of electron-hole pair through an intermediate deep defect states that are, most probably, localized at the barier-nanoisland heterointerface. In our case, the cation vacancies may serve as actual defect states. Firstly, the cation vacancy formation features $\mathrm{A}^{2} \mathrm{~B}^{6}$ semiconductors, and they cause a series of absorption and radiation bands in these materials [11]. Secondly, the deep traps for electrons were found in $\mathrm{CdSe} /$ ZnSe structures with QDs by DLTS method [12]. And thirdly, it should be noted that in the case of the high density of QDs, when the distances between the islands are comparable with their size (for 1.5 ML the density of islands is $\sim 10^{11} \mathrm{~cm}^{-2}$, and the average distance between them is $15-20 \mathrm{~nm}[1])$, an elastic interaction between the islands becomes essential as a result of penetration of nonuniform strain field caused by the QDs into the substrate. This may lead to the gettering effect from QDs and barrier layers with the localization of defects at the interface.

If the excitation is resonant with the low energy wing of the QDs emission band ( $E_{\text {exc }}=2.54 \mathrm{eV}$, Fig. 1(b)), the PL is observed both in Stokes and anti-Stokes spectral ranges. In the Stokes range the band with the maximum position at $\sim 2.522 \mathrm{eV}$ and half-width $\sim 5 \mathrm{meV}$ is observed. We ascribe it to the exciton recombination within the resonantly excited ensemble of the QDs of definite size and/or composition. Its low half-width and independence of its spectral position on the excitation power support the excitonic nature of this band. Using the known de- pendence of the energy of the catodoluminescence band on the size and $\mathrm{Cd}$ content of the islands that was obtained in the framework of the quantum disc model [13], we have estimated the concentration of $\mathrm{Cd}$ corresponding to the emission band $2.522 \mathrm{eV}$. This concentration, $x \approx 0.4$, is noticeably larger than that in the samples grown by the less equilibrium mode of the usual MBE deposition $[1,2]$. The fact that the $2.522 \mathrm{eV}$ band is not observed at high-energy non-resonant excitation (Fig. 1(a)) can be caused by the insufficient density of QDs or by the peculiarities of nonequilibrium carriers relaxation in the system of QDs with spatially inhomogeneous potential.

We would like to note that the efficiency of the UPL at the resonant excitation (Fig. 1(b)) is by an order of magnitude lager than at nonresonant excitation (Fig. $1(\mathrm{c}, \mathrm{d})$ ). This increase of UPL can be caused by the additional contribution of the quantum levels of the QDs that play the role of intermediate levels.

To confirm our assumption concerning UPL excitation, we have investigated the dependence of the UPL intensity on the excitation power. The results shown in Fig. 2 for the excitation with the energy $E_{\text {exc }}=2.41 \mathrm{eV}$ gives the coefficient of the line slope near $\sim 2$. At the

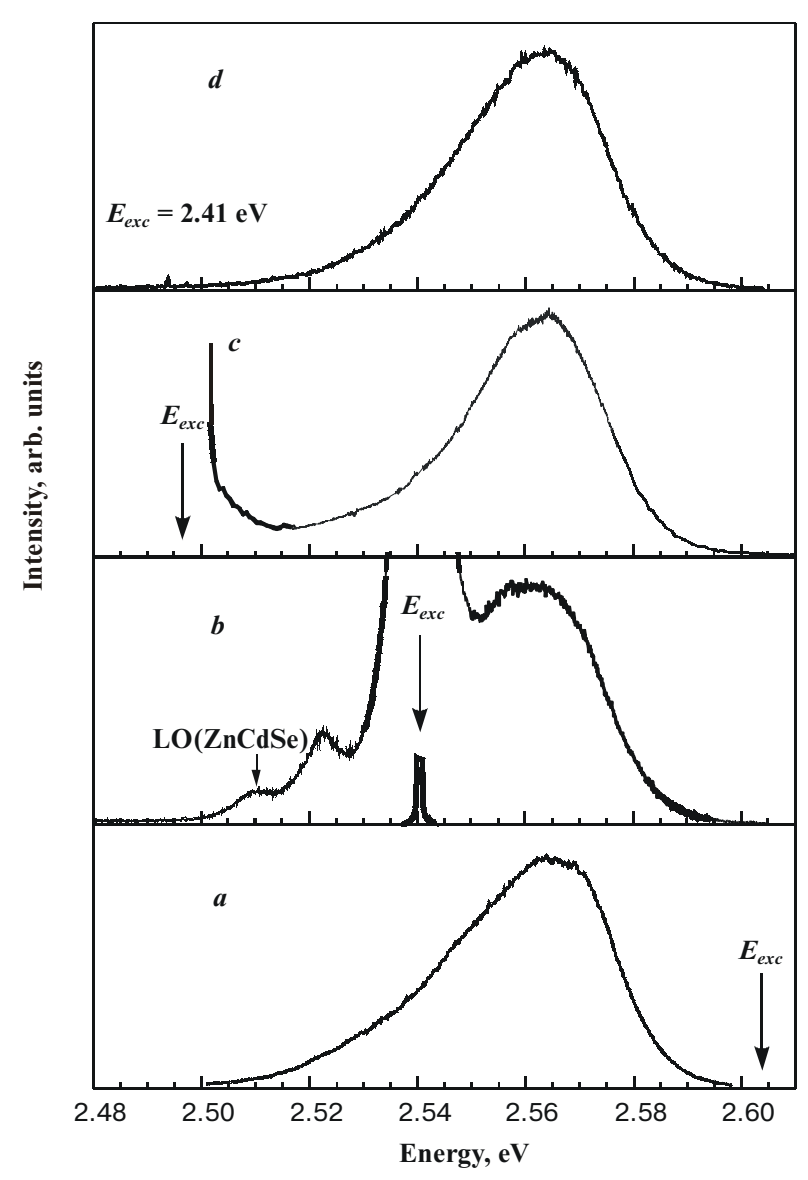

Fig. 1. PL spectra of the $\mathrm{CdSe} / \mathrm{ZnSe}$ nanostructure with the single CdSe insert of the nominal thickness 1.5ML: $a-E_{\text {exc }}=2.60 \mathrm{eV}$; $b-E_{\text {exc }}=2.54 \mathrm{eV} ; c-E_{\text {exc }}=2.497 \mathrm{eV} ; d-E_{\text {exc }}=2.41 \mathrm{eV} . T=90 \mathrm{~K}$. 


\section{V.V. Strelchuk: High-efficient up-conversion of ...}

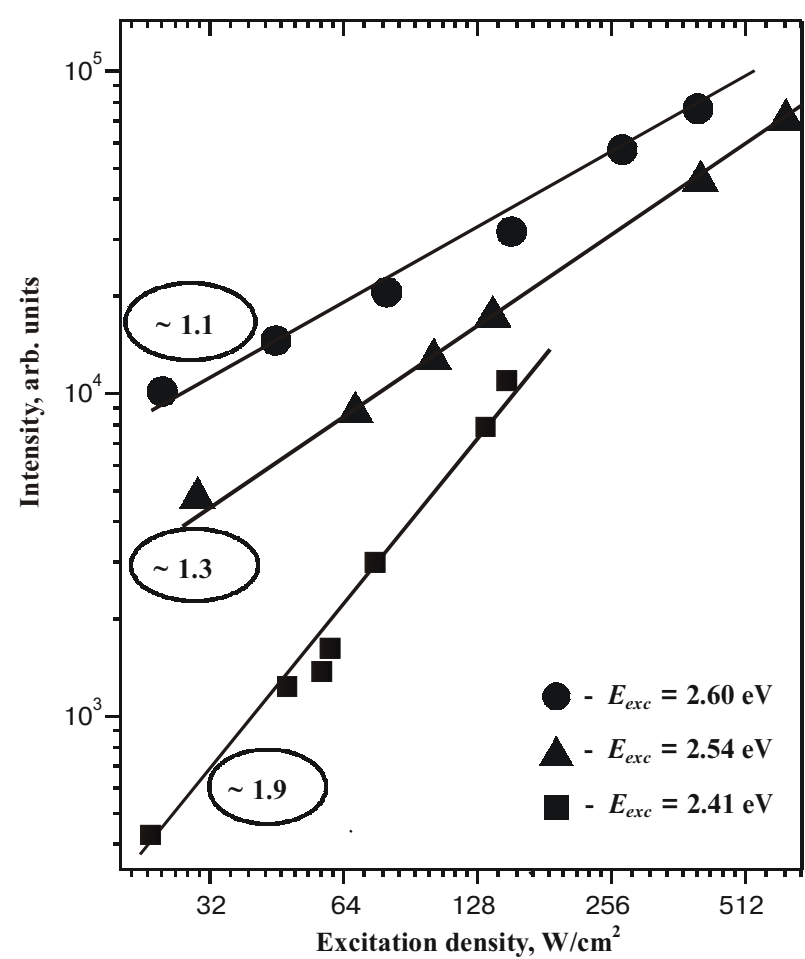

Fig. 2. Power dependence of PL intensity of the $\mathrm{CdSe} / \mathrm{ZnSe}$ (1.5ML) structure at different energy of excitation quanta. Circles and squares, (triangles) correspond to the Stokes $\left(E_{\text {exc }}=\right.$ $=2.60 \mathrm{eV})$ and anti-Stokes $\left(E_{\text {exc }}=2.41 \mathrm{eV}(2.54 \mathrm{eV})\right)$ excitation The slope of each dependence is indicated. $T=90 \mathrm{~K}$. Doublelogarithmic scale is used.

usual high-energy excitation $\left(E_{\text {exc }}=2.60 \mathrm{eV}\right)$, the dependence of PL on the excitation density was almost linear (Fig. 2) that is peculiar for the excitonic recombination mechanism. It should be emphasized that the UPL efficiency at the excitation energy $2.41 \mathrm{eV}$ makes up several percents in comparison to the power-commensurable $\left(P_{\text {exc }} \sim 10 \mathrm{~W} / \mathrm{cm}^{2}\right)$ direct excitation with energy of $2.707 \mathrm{eV}$, i.e., we have a sufficiently intensive process.

Other possible mechanism of the UPL enhancement under resonant excitation (Fig. 1(b)) may be the Auger recombination process. But in this case the cubic-like dependence of intensity on the excitation power is expected, which contradicts our results $\left(\sim I^{1.3}\right.$, Fig. 2$)$. Besides the power of the excitation was low enough $\left(P_{\text {exc }}<400 \mathrm{~W} / \mathrm{cm}^{2}\right)$ for the contribution of the direct two-photon absorption to be insignificant. Therefore, we conclude that in our case the Auger process is not the dominating mechanism of the anti-Stokes emission.

Inasmuch as at low temperature ( $5 \mathrm{~K}$ ) the $\mathrm{ZnSe}$ barrier band gap is $\sim 2.8 \mathrm{eV}$, the UPL was also observed at the excitation with quantum energy $2.41 \mathrm{eV}$, the energy level participating in TS-TPA is situated, at least, $\sim 0.4 \mathrm{eV}$ lower (higher) than conduction (valence) band edge of ZnSe barrier.

UPL was also observed in the sample with the single CdSe inserts of the thickness equal to $0.6 \mathrm{ML}$.
As an alternative of the considered UPL excitation, we analyzed the possibility involving the electronic transitions on the GaAs/ZnSe interface. We took into account that energy positions of GaAs and $\mathrm{ZnSe}$ conduction bands are close (while the top of the GaAs valence band is by $1 \mathrm{eV}$ higher than that of $\mathrm{ZnSe}$ ); due to this fact the electron excited from the GaAs valence band can enter the conductivity band of $\mathrm{ZnSe}$ and then, after diffusion, it can be transferred to the CdSe well. A hole in the CdSe well can appear due to the photoexcitation of an electron into the valence band of GaAs.

But there are some considerations that contradict to the above scenarios:

1. The lifetime of the photoexcited carriers in the direct band GaAs is rather low, therefore the needed intensity of two-step photoexcitation must be rather high to overcome the competing channels of radiative and nonradiative recombination in GaAs itself. But in our case, the UPL was observed at $P<10 \mathrm{~W} / \mathrm{cm}^{2}$.

2. We did not observe even weak UPL of the $\mathrm{ZnSe}$ barrier that is quite possible at this alternative process.

3. We did not observe the plasmon-phonon effects in Raman scattering spectra of GaAs substrate, even when the photoexcitation power was increased by one or two orders of magnitude.

Thus, the low-temperature UPL was revealed in CdSe/ $\mathrm{ZnSe}$ heterostructures with the single CdSe insert of the nominal thickness of $1.5 \mathrm{ML}$, forming $\mathrm{Cd}_{\mathrm{x}} \mathrm{Zn}_{1-\mathrm{x}}$ Se quantum dots. This UPL is characterized by the anomalously high efficiency in comparison with the previously observed one in bulk semiconductors and QW structures. We suppose UPL to be connected with the TS-TPA process through the defect states localized at the vicinity of CdSe QDs. Cation vacancies may serve as such defects and the high concentration of the cation vacancies in the case of large density of QDs cause the anomalously high intensity of up-conversion emission.

\section{References}

1. N. Peranio, A. Rosenauer, D. Gerthsen, S.V. Sorokin, I.V. Sedova, S.V. Ivanov, Structural and chemical analysis of $\mathrm{CdSe} / \mathrm{ZnSe}$ nanostructures by transmission electron microscopy //Phys. Rev. B 61, 16015-16024 (2000).

2. D. Litvinov, A. Rosenauer, D. Gerthsen, N.N. Ledentsov, Character of the $\mathrm{Cd}$ in ultrathin CdSe layers in a $\mathrm{ZnSe}$ matrix //Phys. Rev. B 61, 16819-16826 (2000).

3. M.Ya. Valakh, S.V. Ivanov, N. Mestres, J. Pascual, T.V. Shubina, S.V. Sorokin, V.V. Streltchuk, G. Pozina, B. Monemar, Optical investigation of $\mathrm{CdSe} / \mathrm{ZnSe}$ quantum nanostructures //Semicond. Sci. Technolog. 17 173-177 (2002).

4. L.V. Borkovska, M.Ya. Valakh, E. F. Venger, Yu.G. Sadofyev, N.O. Korsunska, V.V. Strelchuk, G.N. Semenova, M.V. Vuychik, Investigation of inhomogeneous broadening of $\mathrm{CdSe} / \mathrm{ZnSe}$ nanoisland photoluminescence band by resonant excitation methods // Physica E (2003) (in press).

5. D.A. Mazurenko, A.V. Scherbakov, A.V. Akimov, A.J. Kent, M. Henini, Enchancement of luminescence intensity induced by $1.06 \mathrm{~mm}$ excitation in InAs/GaAs quantum dots //Semicond. Sci. Technolog. 14 1132-1135 (1999).

6. T. Kita, T. Nishino, C. Geng, F. Scholz, H. Schweizer, Dynamic process of anti-Stokes photoluminescence at a long- 
V.V. Strelchuk: High-efficient up-conversion of ...

range-ordered $\mathrm{Ga}_{0.5} \mathrm{In}_{0.5} \mathrm{P} / \mathrm{GaAs}$ heterointerface //Phys. Rev. B 59, 15358-15362 (1999).

7. P.P. Paskov, P.O. Holtz, B. Monemar, J.M. Garcia, W.V. Schoenfeldand, P.M. Petroff, Optical up-conversion processes in InAs quantum dots //Jpn. J. Appl. Phys. 40(3B), 20802083 (2001)

8. I.V. Ignatiev, I.E. Kozin, H.W. Ren, S. Sugou, Y. Masumoto, Anti-Stokes photoluminescence of InP self-assembled quantum dots in the presence of electric current //Phys. Rev. B $\mathbf{6 0}$, R14001-R14004 (1999).

9. S.V. Ivanov, A.A. Toropov, T.V. Shubina, S.V. Sorokin, A.V. Lebedev, I.V. Sedova, P.S. Kop'ev, G.R. Pozina, J.P. Bergman, B. Monemar, Growth and excitonic properties of single fractional monolayer $\mathrm{CdSe} / \mathrm{ZnSe}$ structures //J. Appl. Phys. 83, 3168-3171 (1998).
10. C.S. Kim, M. Kim, S. Lee, J.K. Furdyna, M. Dobrowolska, H. Rho, L.M. Smith, H.E. Jackson, Optical observation of quantum-dot formation in sub-critical CdSe layers grown on ZnSe //J. Cryst. Growth, 214/215, 761-764 (2000).

11. K.M. Lee, Le Si Dang, G.D. Watkins, Optically detected magnetic resonance of the zinc vacancy in $\mathrm{ZnSe} / /$ Solid State Commun. 35, 527-530 (1980).

12. V.G. Litvinov, V.I. Kozlovsky, Yu.G. Sadofyev, Deep-level transient spectroscopy and cathodoluminescence of CdSe/ ZnSe QD structures grown on GaAs (100) by MBE //Phys. Stat. Sol. (b) 229, 513-517 (2002).

13. I. Yamakawa, S. Sorokin, A. Toropov, S. Ivanov, A. Nakamura. Catodoluminescence studi of quantum-size and alloying effects in single fractional monolayer $\mathrm{CdSe} / \mathrm{ZnSe}$ structures //Jpn. J. Appl. Phys. 39(8B), L851-L854 (2000). 